\title{
Wideband and flat-gain amplifier based on high concentration erbium- doped fibres in parallel double-pass configuration
}

\author{
B.A. Hamida, X.S. Cheng, S.W. Harun, A.W. Naji, H. Arof, W. Al-Khateeb, S. Khan, H. Ahmad
}

\begin{abstract}
A wideband and flat gain erbium-doped fibre amplifier (EDFA) is demonstrated using a hybrid gain medium of a zirconiabased erbium-doped fibre (Zr-EDF) and a high concentration erbium-doped fibre (EDF). The amplifier has two stages comprising a 2-m-long ZEDF and 9-m-long EDF optimised for $\mathrm{C}$ - and L-band operations, respectively, in a double-pass parallel configuration. A chirp fibre Bragg grating (CFBG) is used in both stages to ensure double propagation of the signal and thus to increase the attainable gain in both $\mathrm{C}$ - and $\mathrm{L}$-band regions. At an input signal power of $0 \mathrm{dBm}$, a flat gain of $15 \mathrm{~dB}$ is achieved with a gain variation of less than $0.5 \mathrm{~dB}$ within a wide wavelength range from 1530 to $1605 \mathrm{~nm}$. The corresponding noise figure varies from 6.2 to $10.8 \mathrm{~dB}$ within this wavelength region.
\end{abstract}

Keywords: double-pass amplifier, wideband amplifier, zirconia-based erbium-doped fibre.

\section{Introduction}

The tremendous growth of the internet and data traffic has created an enormous demand for broadband dense wavelength-division-multiplexed (DWDM) optical communication systems. Since the silica-based transmission fibres have a wideband operating window ranging from 1400-1700 nm, optical amplifiers with a wider amplification bandwidth are required to cover the full range of the DWDM systems [1-3]. To extend the wavelength range, several glass hosts such as tellurite [4], multicomponent silicate [5], bismuth oxide based glass [6-9] and zirconia-oxide based glass [10] have been developed for an erbium-doped optical amplifier (EDFA). Recently, a zirconia-based erbium-doped fibre (Zr-EDF) with a highly doped erbium ion concentration has been introduced for realising an efficient and compact optical amplifier, where the combination of both $\mathrm{Zr}$ and $\mathrm{Al}$ ions is used to achieve

B.A. Hamida, A.W. Naji, W. Al-Khateeb, S. Khan Optoelectronics Laboratory, Electrical and Computer Engineering Department, Faculty of Engineering, International Islamic University Malaysia (IIUM), 53100 Gombak, Kuala Lumpur, Malaysia;

X.S. Cheng, H.Arof Department of Electrical Engineering, Faculty of Engineering, University of Malaya, 50603 Kuala Lumpur Malaysia;

H. Ahmad Photonics Research Center, University of Malaya, 50603 Kuala Lumpur, Malaysia;

S.W. Harun Department of Electrical Engineering, Faculty of

Engineering, University of Malaya, 50603 Kuala Lumpur Malaysia; Photonics Research Center, University of Malaya, 50603 Kuala Lumpur, Malaysia; e-mail: swharun@um.edu.my

Received 15 July 2011; revision received 8 October 2011

Kvantovaya Elektronika 42 (3) 241-243 (2012)

Submitted in English a high erbium doping concentration of $2800 \mathrm{ppm}$ in the glass host without any phase separations of rare-earths [11]. A highly doped silica based erbium-doped fibre (EDF) with an erbium ion concentration of 2200 ppm is also commercially available [8].

In this paper, a hybrid wideband optical amplifier with a flat-gain characteristic is proposed using a combination of $\mathrm{Zr}-\mathrm{EDF}$ and EDF as the gain medium. The amplifier consists of two stages where $\mathrm{Zr}-\mathrm{EDF}$ and EDF operating in the C-band and L-band, respectively, are used. The performance of the amplifier is investigated in both linear and parallel configurations. A chirp fibre Bragg grating (CFBG) is incorporated in each stage to allow the double-pass operation. A flatgain output is achieved with the parallel configuration, in which a C-band Zr-EDF amplifier is combined with an L-band EDF amplifier using a C/L-band wavelength division multiplexing (WDM) coupler for multiplexing and demultiplexing the channels in the 1525-1565-nm and 1570-1615nm wavelength regions.

\section{Experimental}

Figure 1a shows the proposed wideband EDFA in a doublepass parallel configuration. At the input of the optical amplifier, a C/L-band WDM coupler is used to separate the WDM signals to $\mathrm{C}$ - and L-bands. The C- and L-band signals are amplified by a 2-m-long Zr-EDF and a 9-m-long EDF, which are forward pumped by a $980 \mathrm{~nm}$ and $1480 \mathrm{~nm}$ laser diode, respectively. The Zr-EDF used is drawn from a fibre perform. The preform was fabricated using, in a ternary glass host, zirconia-yttria-aluminum codoped silica fibre through a solution doping process along with modified chemical vapour deposition (MCVD). The peak absorption of the Zr-EDF at $978 \mathrm{~nm}$ was measured to be $14.5 \mathrm{~dB} \mathrm{~m}^{-1}$, which is equivalent to the erbium ions concentration of $2800 \mathrm{ppm}$. The EDF used has an erbium ion concentration of about $2200 \mathrm{ppm}$. At the end of each stage, a broadband CFBG operating at a C- or $\mathrm{L}$-band is incorporated to reflect the C- or L-band signals for double-pass operation. These signals are combined together by the C/L-band WDM coupler at the input port of the amplifier before they are routed to the output port via optical circulator (see Fig. 1a). The insertion loss of the WDM couplers is assumed to be 0.9 and $1.8 \mathrm{~dB}$ in the $\mathrm{C}$ - and L-band region, respectively.

The performance of a hybrid amplifier with a serial configuration (Fig. 1b) is also investigated for comparison purpose. The serial amplifier uses similar components as those of the proposed parallel amplifier (Fig. 1a). The C-band CFBG is placed in between the two stages to act as a reflector for the C-band EDFA. It reflects the C-band signal for double-pass 